\title{
Pairwise interactive knowledge and Nash equilibrium
}

Citation for published version (APA):

Bach, C. W., \& Tsakas, E. (2012). Pairwise interactive knowledge and Nash equilibrium. METEOR, Maastricht University School of Business and Economics. METEOR Research Memorandum No. 008 https://doi.org/10.26481/umamet.2012008

Document status and date:

Published: 01/01/2012

DOI:

10.26481/umamet.2012008

Document Version:

Publisher's PDF, also known as Version of record

\section{Please check the document version of this publication:}

- A submitted manuscript is the version of the article upon submission and before peer-review. There can be important differences between the submitted version and the official published version of record.

People interested in the research are advised to contact the author for the final version of the publication, or visit the DOI to the publisher's website.

- The final author version and the galley proof are versions of the publication after peer review.

- The final published version features the final layout of the paper including the volume, issue and page numbers.

Link to publication

\footnotetext{
General rights rights.

- You may freely distribute the URL identifying the publication in the public portal. please follow below link for the End User Agreement:

www.umlib.nl/taverne-license

Take down policy

If you believe that this document breaches copyright please contact us at:

repository@maastrichtuniversity.nl

providing details and we will investigate your claim.
}

Copyright and moral rights for the publications made accessible in the public portal are retained by the authors and/or other copyright owners and it is a condition of accessing publications that users recognise and abide by the legal requirements associated with these

- Users may download and print one copy of any publication from the public portal for the purpose of private study or research.

- You may not further distribute the material or use it for any profit-making activity or commercial gain

If the publication is distributed under the terms of Article $25 \mathrm{fa}$ of the Dutch Copyright Act, indicated by the "Taverne" license above, 


\section{Maastricht University}

Christian W. Bach, Elias Tsakas

Pairwise interactive knowledge and Nash equilibrium

$\mathrm{RM} / 12 / 008$

\section{METEOR}

Maastricht University School of Business and Economics

Maastricht Research School of Economics

of Technology and Organization

P. B. Box 616

NL -6200 MD Maastricht

The Netherlands 


\title{
PAIRWISE INTERACTIVE KNOWLEDGE AND NASH EQUILIBRIUM
}

\section{Christian W. Bach and Elias Tsakas}

\begin{abstract}
We provide epistemic conditions for Nash equilibrium, which are considerably weaker than the standard ones by Aumann and Brandenburger (1995). Indeed, we simultaneously replace common knowledge of conjectures and mutual knowledge of rationality with strictly weaker epistemic conditions of pairwise common knowledge of conjectures and pairwise mutual knowledge of rationality respectively. It is also shown that, unlike the Aumann and Brandenburger's conditions, ours do not imply common knowledge of rationality. Surprisingly, they actually do not even imply mutual knowledge of rationality.

Keywords: Nash equilibrium, pairwise common knowledge, pairwise mutual knowledge, rationality, conjectures, epistemic game theory.
\end{abstract}

\section{INTRODUCTION}

In their seminal paper, Aumann and Brandenburger (1995) provided epistemic conditions for Nash equilibrium. Accordingly, if there exists a common prior, then mutual knowledge of rationality and common knowledge of each player's conjecture about the opponents' strategies imply Nash equilibrium in normal form games with more than two players. As they pointed out, in their epistemic conditions common knowledge enters the picture in an unexpected way; in fact, they stressed that what is needed is common knowledge of the players' conjectures and not of the players' rationality (Aumann and Brandenburger, 1995, p. 1163). Their result challenged the widespread view that common knowledge of rationality is essential for Nash equilibrium. Subsequently, Polak (1999) showed that in complete information games, Aumann and Brandenburger's conditions actually do imply common knowledge of rationality. In a sense, his result thus restored some of the initial belief in the importance of common knowledge of rationality for Nash equilibrium.

Here, we introduce weaker epistemic conditions for Nash equilibrium than Aumann and Brandenburger (1995), by simultaneously relaxing their two main assumptions. Our new conditions are based on imposing pairwise mutual knowledge of rationality and pairwise common knowledge of conjectures only for some pairs of players. This constitutes a significant weakening of Aumann and Brandenburger's epistemic foundation, as their conditions correspond to pairwise mutual knowledge of rationality and pairwise common knowledge of conjectures for all pairs of players. Note that this difference is particularly important for large games, such as economies with many agents.

Apart from generalizing Aumann and Brandenburger's standard result, we also contribute to the debate about the connection between common knowledge of rationality and Nash equilibrium. Indeed, we prove that - contrary to what Polak (1999) showed for Aumann and Brandenburger's foundation - 
our conditions do not entail common knowledge of rationality. Surprisingly, they actually do not even imply mutual knowledge of rationality.

\section{PRELIMINARIES}

\subsection{Games}

Let $\Gamma=\left(I,\left(S_{i}\right)_{i \in I},\left(U_{i}\right)_{i \in I}\right)$ be game in normal form, where $I=\{1, \ldots, N\}$ denotes the finite set of players with typical element $i$, and $S_{i}$ denotes the finite set of pure strategies, also called choices, with typical element $s_{i}$ for every player $i \in I$. Moreover, define $S:=\times_{i \in I} S_{i}$ with typical element $s=\left(s_{1}, \ldots, s_{N}\right)$ and $S_{-i}:=\times_{j \in I \backslash\{i\}} S_{j}$ with typical element $s_{-i}=\left(s_{1}, \ldots, s_{i-1}, s_{i+1}, \ldots, s_{N}\right)$. Then, the function $U_{i}: S_{i} \times S_{-i} \rightarrow \mathbb{R}$ denotes player $i$ 's utility function.

A probability measure $\mu_{i} \in \Delta\left(S_{-i}\right)$ on the set of the opponents' choice combinations is called a conjecture of $i$, where $\mu_{i}\left(s_{-i}\right)$ signifies the probability that $i$ attributes to the opponents playing $s_{-i}$. Slightly abusing notation, let $\mu_{i}\left(s_{j}\right):=\operatorname{marg}_{S_{j}} \mu_{i}\left(s_{j}\right)$ denote the probability that $i$ assigns to $j$ playing $s_{j}$. Note that it is standard to admit correlated beliefs, i.e. $\mu_{i}$ is not necessarily a product measure, hence the probability $\mu_{i}\left(s_{1}, \ldots, s_{i-1}, s_{i+1}, \ldots, s_{N}\right)$ can differ from the product $\mu_{i}\left(s_{1}\right) \cdots \mu_{i}\left(s_{i-1}\right) \mu_{i}\left(s_{i+1}\right) \cdots \mu_{i}\left(s_{N}\right)$ of the marginal probabilities. ${ }^{1}$ Given a conjecture $\mu_{i} \in \Delta\left(S_{-i}\right)$, player $i$ 's expected utility from playing some strategy $s_{i} \in S_{i}$ is given by

$$
u_{i}\left(s_{i}, \mu_{i}\right):=\sum_{s_{-i} \in S_{-i}} \mu_{i}\left(s_{-i}\right) U_{i}\left(s_{i}, s_{-i}\right) .
$$

We say that a strategy $s_{i}$ is a best response to $\mu_{i}$, and write $s_{i} \in B R_{i}\left(\mu_{i}\right)$, whenever $u_{i}\left(s_{i}, \mu_{i}\right) \geq u_{i}\left(s_{i}^{\prime}, \mu_{i}\right)$ for all $s_{i}^{\prime} \in S_{i}$.

A randomization over a player's pure strategies is called mixed strategy, and is typically denoted by $\sigma_{i} \in \Delta\left(S_{i}\right)$ for all $i \in I$. Let $\Delta\left(S_{1}\right) \times \cdots \times \Delta\left(S_{N}\right)$ denote the space of mixed strategy profiles, with typical element $\left(\sigma_{1}, \ldots, \sigma_{N}\right)$. Slightly abusing terminology, we say that a pure strategy $s_{i} \in S_{i}$ is a best response to $\sigma$, and write $s_{i} \in B R_{i}(\sigma)$, whenever $s_{i}$ is a best response to the product measure $\sigma_{-i}:=\operatorname{marg}_{S_{-i}} \sigma$, which is an element of $\Delta\left(S_{1}\right) \times \cdots \times \Delta\left(S_{i-1}\right) \times \Delta\left(S_{i+1}\right) \times \cdots \times \Delta\left(S_{N}\right) \subseteq \Delta\left(S_{-i}\right)$. Nash's notion of equilibrium can then be defined as follows: a mixed strategy profile $\left(\sigma_{1}, \ldots, \sigma_{N}\right)$ is a Nash equilibrium of the game $\Gamma$, whenever $s_{i} \in B R_{i}(\sigma)$ for all $s_{i} \in \operatorname{supp}\left(\sigma_{i}\right)$ and for all $i \in I$.

\footnotetext{
${ }^{1}$ Intuitively, a player's belief on his opponents' choices can be correlated, even though players choose independently from each other.
} 


\subsection{Epistemic Models}

The epistemic approach to game theory analyzes the relation between knowledge, belief, and choice of rational players. While classical game theory is based on the two basic primitives - game form and choice - epistemic game theory adds an epistemic framework as a third elementary component so that knowledge and beliefs can be explicitly modelled in games.

Here, we follow Aumann's approach to epistemic game theory and use the partitional model introduced by Aumann (1976). Formally, an epistemic model of some game $\Gamma$ is a tuple $\mathcal{A}^{\Gamma}=\left(\Omega, \pi,\left(\mathcal{P}_{i}\right)_{i \in I},\left(\hat{s}_{i}\right)_{i \in I}\right)$, also called Aumann model of $\Gamma$, consisting of a finite set $\Omega$ of possible worlds, also called states, with typical element $\omega$, together with a full support common prior $\pi \in \Delta(\Omega)$. Furthermore, every player $i \in I$ is endowed with an information partition $\mathcal{P}_{i}$ of $\Omega$, as well as a choice function $\hat{s}_{i}: \Omega \rightarrow S_{i}$. The cell of $\mathcal{P}_{i}$ containing the world $\omega$ is denoted by $P_{i}(\omega)$ and contains all worlds considered possible by $i$ at $\omega$. Besides, a set $E \subseteq \Omega$ of possible worlds is called event. Knowledge is formalized in terms of events: the set of states at which agent $i$ knows $E$ is defined as

$$
K_{i}(E):=\left\{\omega \in \Omega: P_{i}(\omega) \subseteq E\right\}
$$

Then, it is said that $i$ knows $E$ at $\omega$, whenever $\omega \in K_{i}(E)$. For every player $i \in I$ his choice function $\hat{s}_{i}$ specifies his pure strategy at each world and is assumed to be $\mathcal{P}_{i}$-measurable, i.e. $\hat{s}_{i}\left(\omega^{\prime}\right)=\hat{s}_{i}(\omega)$ for all $\omega^{\prime} \in P_{i}(\omega)$, which implies that $i$ knows his own strategy. Note that $\hat{s}_{i}$ induces a coarsening of $\mathcal{P}_{i}$ consisting of events of the form $\left[s_{i}\right]:=\left\{\omega \in \Omega: \hat{s}_{i}(\omega)=s_{i}\right\}$.

An event is mutually known if everyone knows it. Formally, $E \subseteq \Omega$ is mutual knowledge at $\omega$, whenever $\omega \in K(E)$, where

$$
K(E):=\bigcap_{i \in I} K_{i}(E)
$$

Iterating the mutual knowledge operator then yields higher-order mutual knowledge. Formally, $m$ order mutual knowledge of $E$ is inductively defined by $K^{m}(E):=K\left(K^{m-1}(E)\right)$ for all $m>0$, with $K^{1}(E):=K(E)$. Then, an event $E$ is commonly known whenever everyone knows $E$, everyone knows that everyone knows $E$, etc. Formally, common knowledge of $E$ can then be stated as

$$
C K(E):=\bigcap_{m>0} K^{m}(E) .
$$

Aumann (1976) introduced an alternative yet formally equivalent definition of common knowledge in terms of the finest common coarsening of the players' information partitions, also called the meet. Formally, let $\mathcal{M}:=\mathcal{P}_{1} \wedge \cdots \wedge \mathcal{P}_{N}$ denote the meet, with $M(\omega)$ being the element of $\mathcal{M}$ that contains 
$\omega$. It can be shown that

$$
C K(E)=\{\omega \in \Omega: M(\omega) \subseteq E\}
$$

Given an epistemic model $\mathcal{A}^{\Gamma}$, for every player $i \in I$ conjectures about the opponents' choices can be derived at every world $\omega \in \Omega$ from the common prior. Formally, for every world $\omega \in \Omega$ and for every $s_{-i} \in S_{-i}$, the probability that $i$ 's conjecture assigns to $s_{-i}$ is defined as

$$
\hat{\mu}_{i}(\omega)\left(s_{-i}\right):=\pi\left(\left[s_{-i}\right] \mid P_{i}(\omega)\right)
$$

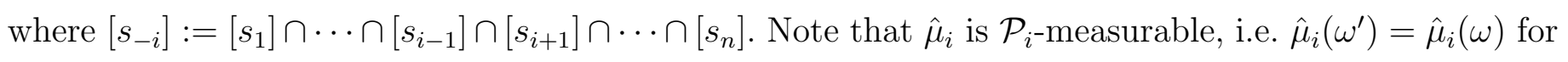
all $\omega^{\prime} \in P_{i}(\omega)$. Henceforth, let

$$
\left[\mu_{i}\right]:=\left\{\omega \in \Omega: \hat{\mu}_{i}(\omega)=\mu_{i}\right\}
$$

denote the event that player $i$ entertains conjecture $\mu_{i}$.

Furthermore, player $i$ is rational at world $\omega$, whenever $\hat{s}_{i}(\omega) \in B R_{i}\left(\hat{\mu}_{i}(\omega)\right)$. Let

$$
R_{i}:=\left\{\omega \in \Omega: \hat{s}_{i}(\omega) \in B R_{i}\left(\hat{\mu}_{i}(\omega)\right)\right\}
$$

denote the event that $i$ is rational. Rationality of all players is then given by the event

$$
R:=\bigcap_{i \in I} R_{i}
$$

\subsection{Aumann and Brandenburger's Epistemic Conditions for Nash equilibrium}

In their seminal paper, Aumann and Brandenburger (1995) provide epistemic conditions for Nash equilibrium. ${ }^{2}$ Accordingly, if conjectures are derived from a common prior and are commonly known, while at the same time rationality as well as the utility functions are mutual knowledge, then all players different from $i$ entertain the same marginal conjecture about $i$ 's choice, and the marginal conjectures constitute a Nash equilibrium of the game. Fixing common knowledge of the game as an implicit background assumption, Aumann and Brandenburger's epistemic foundation for Nash equilibrium can formally be stated as follows.

TheOREM 1 (Aumann and Brandenburger (1995)) Let $\Gamma$ be a game and $\mathcal{A}^{\Gamma}$ be an epistemic model of it. Suppose that at some world $\omega \in \Omega$ there exists a tuple $\left(\mu_{1}, \ldots, \mu_{N}\right)$ of conjectures such that $\omega \in K(R) \cap C K\left(\bigcap_{i \in I}\left[\mu_{i}\right]\right)$. Then, there exists a mixed strategy profile $\left(\sigma_{1}, \ldots, \sigma_{N}\right)$ such that

\footnotetext{
${ }^{2}$ Note that Aumann and Brandenburger (1995) employ the formalism of type structures, which is notationally distinct yet formally equivalent to Aumann models.
} 
(i) $\operatorname{marg}_{S_{i}} \mu_{j}=\sigma_{i}$ for all $j \in I \backslash\{i\}$,

(ii) $\left(\sigma_{1}, \ldots, \sigma_{N}\right)$ is a Nash equilibrium of $\Gamma$.

Subsequently, Polak (1999) shows that common knowledge of conjectures and mutual knowledge of rationality imply common knowledge of rationality. In the context of Theorem 1, Polak's result implies that sufficient conditions for Nash equilibrium without common knowledge of rationality need to relax common knowledge of conjectures or mutual knowledge of rationality. In fact, we will weaken both assumptions in Section 4 below and yet obtain Nash equilibrium.

\section{PAIRWISE INTERACTIVE KNOWLEDGE}

The standard intuitive explanation for the emergence of common knowledge is based on public announcement. Accordingly, once an event is publicly announced it becomes common knowledge in the sense that not only everyone knows it, but also everyone knows that everyone knows it, etc. Note that for mutual knowledge to obtain, the agents are only required to each know the event, and hence mere private announcements suffice.

Yet, an event may be publicly (privately) announced to some but not all players. For instance, an event could be publicly (privately) announced to Alice and Bob, but not to Claire. Common knowledge (mutual knowledge) of the event between Alice and Bob would then emerge, but not necessarily common knowledge (mutual knowledge). Due to such epistemic possibilities we now introduce pairwise interactive knowledge operators.

Let $E \subseteq \Omega$ be some event and $i, j \in I$ be two players. We say that $E$ is pairwise mutual knowledge between $i$ and $j$ whenever they both know $E$. Formally, pairwise mutual knowledge of $E$ between $i$ and $j$ is denoted by the event

$$
K_{i, j}(E):=K_{i}(E) \cap K_{j}(E) .
$$

Note that mutual knowledge implies pairwise mutual knowledge, but not conversely. We say that $E$ is pairwise common knowledge between $i$ and $j$ whenever $E$ is commonly known between them. Formally, let $\mathcal{M}_{i, j}:=\mathcal{P}_{i} \wedge \mathcal{P}_{j}$ with $M_{i, j}(\omega)$ denoting the element of $\mathcal{M}_{i, j}$ that contains $\omega$. Pairwise common knowledge of $E$ between $i$ and $j$ is then defined as the event

$$
C K_{i, j}(E):=\left\{\omega \in \Omega: M_{i, j}(\omega) \subseteq E\right\}
$$

Observe that, as $\mathcal{M}_{i, j}$ is a refinement of $\mathcal{M}$, common knowledge implies pairwise common knowledge, but not conversely. 
In contrast to the standard notions of mutual and common knowledge, our two pairwise epistemic operators describe interactive knowledge only locally for pairs of agents, postulating the existence of exclusively binary relations of epistemic relevance. Formally, we represent a set of such binary relations by means of an undirected graph $G=(I, \mathcal{E})$, where the set of vertices $I$ denotes the set of players from $\Gamma$, and the set of edges $\mathcal{E}$ describe binary symmetric relations $(i, j) \in I \times I$ between pairs of players.

The graph $G$ does neither enrich the epistemic model nor add any additional structure to the game whatsoever, but only provides a formal framework for expressing pairwise local conditions of interactive knowledge, e.g. a graph containing an edge between $i$ and $j$ but not between $j$ and $k$ can be used to model a situation where an event is pairwise mutual knowledge between $i$ and $j$ but not between $j$ and $k$. Thus, the connectedness of two agents by an edge is of purely epistemic and not physical character. However, $G$ could also be interpreted as a network. For instance, in a large economy agents may have access to information about relevant personal characteristics - such as rationality or conjectures - of their neighbours only.

Next, some graph theoretic notions are recalled. A sequence $\left(i_{k}\right)_{k=1}^{m}$ of players is a path whenever $\left(i_{k}, i_{k+1}\right) \in \mathcal{E}$ for all $k \in\{1, \ldots, m-1\}$, i.e. in a path every two consecutive players are linked by an edge. Moreover, a graph $G$ is called connected if it contains a path $\left(i_{k}\right)_{k=1}^{m}$ such that for every $i \in I$ there is some $k \in\{1, \ldots, m\}$ with $i_{k}=i$. Besides, $G$ is Hamiltonian, whenever there exists a path $\left(i_{k}\right)_{k=1}^{N}$ such that for every $i \in I$ there is a unique $k \in\{1, \ldots, N\}$ with $i_{k}=i$, and also $\left(i_{1}, i_{N}\right) \in \mathcal{E}$. Intuitively, a Hamiltonian graph contains a cycle in which each player appears exactly once. In addition, $G$ is complete, if $(i, j) \in \mathcal{E}$ for all $i, j \in I$.

Two specific pairwise-local epistemic conditions are now introduced.

Definition 1 Let $\Gamma$ be a game, $\mathcal{A}^{\Gamma}$ be an epistemic model of it, $G$ be an undirected graph, $\omega$ be a world, and $\left(\mu_{1}, \ldots, \mu_{N}\right)$ be a tuple of conjectures.

- Rationality is $G$-pairwise mutual knowledge at $\omega$, whenever $\omega \in K_{i, j}\left(R_{i} \cap R_{j}\right)$ for all $(i, j) \in \mathcal{E}$.

- Conjectures are $G$-pairwise common knowledge of conjectures at $\omega$, whenever $\omega \in C K_{i, j}\left(\left[\mu_{i}\right] \cap\left[\mu_{j}\right]\right)$ for all $(i, j) \in \mathcal{E}$.

Note that henceforth an edge between two agents $i$ and $j$ in a graph $G$ signifies that $i$ and $j$ entertain both pairwise mutual knowledge of rationality as well as pairwise common knowledge of conjectures.

The standard notions of mutual knowledge of rationality and common knowledge of conjectures, which are also used by Aumann and Brandenburger (1995), are weakened by G-pairwise mutual knowledge of 
rationality and $G$-pairwise common knowledge of conjectures, respectively. Formally, observe that

$$
\begin{aligned}
K(R) & =\bigcap_{i \in I} K_{i}\left(R_{1} \cap \cdots \cap R_{N}\right) \\
& \subseteq \bigcap_{i \in I} \bigcap_{j \in I:(i, j) \in \mathcal{E}} K_{i, j}\left(R_{1} \cap \cdots \cap R_{N}\right) \\
& \subseteq \bigcap_{i \in I} \bigcap_{j \in I:(i, j) \in \mathcal{E}} K_{i, j}\left(R_{i} \cap R_{j}\right),
\end{aligned}
$$

as well as

$$
C K\left(\bigcap_{i \in I}\left[\mu_{i}\right]\right) \subseteq \bigcap_{i \in I} \bigcap_{j \in I:(i, j) \in \mathcal{E}} C K_{i, j}\left(\left[\mu_{i}\right] \cap\left[\mu_{j}\right]\right) .
$$

Indeed, our two concepts are considerably weaker than the standard notions on two distinct dimensions. Firstly, the events rationality and conjectures in Definition 1 only refer to the rationality and the conjectures, respectively, of the two connected agents. Secondly, our two pairwise-local epistemic conditions impose epistemic restrictions only on the pairs of connected players in the graph, whereas standard interactive knowledge does so across all pairs of players. In fact, mutual knowledge and common knowledge coincide with $G$-mutual knowledge and $G$-common knowledge, respectively, whenever $G$ is complete.

The following example illustrates the two new concepts of $G$-pairwise mutual knowledge of rationality and $G$-pairwise common knowledge of conjectures and also relates them to the standard notions of mutual knowledge of rationality and common knowledge of conjectures.

EXAMPLE 1 Consider the asymmetric coordination game $\Gamma=\left(I,\left(S_{i}\right)_{i \in I},\left(U_{i}\right)_{i \in I}\right)$, where $I=\{$ Alice, Bob, Claire, Donald $\}, S_{i}=\{h, \ell\}$ for all $i \in I$, and

$$
U_{i}\left(s_{\text {Alice }}, s_{\text {Bob }}, s_{\text {Claire }}, s_{\text {Donald }}\right)= \begin{cases}2 & \text { if } s_{i}=h \text { for all } i \in I, \\ 1 & \text { if } s_{i}=\ell \text { for all } i \in I, \\ 0 & \text { otherwise }\end{cases}
$$

Now, suppose that an epistemic model $\mathcal{A}^{\Gamma}$ of $\Gamma$ is given by

$$
\begin{aligned}
\Omega & =\left\{\omega_{1}, \omega_{2}, \omega_{3}\right\} \text { endowed with a uniform common prior } \pi \\
\mathcal{P}_{\text {Alice }} & =\left\{\left\{\omega_{1}\right\}_{\ell} ;\left\{\omega_{2}, \omega_{3}\right\}_{\ell}\right\} \\
\mathcal{P}_{\text {Bob }} & =\left\{\left\{\omega_{1}\right\}_{\ell} ;\left\{\omega_{2}\right\}_{\ell} ;\left\{\omega_{3}\right\}_{h}\right\} \\
\mathcal{P}_{\text {Claire }} & =\left\{\left\{\omega_{1}, \omega_{2}\right\}_{\ell} ;\left\{\omega_{3}\right\}_{h}\right\} \\
\mathcal{P}_{\text {Donald }} & =\left\{\left\{\omega_{1}\right\}_{\ell} ;\left\{\omega_{2}\right\}_{\ell} ;\left\{\omega_{3}\right\}_{h}\right\}
\end{aligned}
$$


with the information sets' indices denoting the respective player's strategy given by the choice function. Let $G=(I, \mathcal{E})$ be a Hamiltonian graph such that

$$
\begin{aligned}
I & =\{\text { Alice }, \text { Bob }, \text { Claire }, \text { Donald }\} \\
\mathcal{E} & =\{(\text { Alice }, \text { Bob }),(\text { Bob }, \text { Claire }),(\text { Claire }, \text { Donald }),(\text { Donald }, \text { Alice })\} .
\end{aligned}
$$

Observe that the conjectures are $G$-pairwise common knowledge at $\omega_{1}$. Indeed, Alice's and Bob's conjectures - Alice being certain that each of her opponents plays $\ell$ and $B o b$ being certain that each of his opponents plays $\ell$ - are pairwise common knowledge between them. Also, conjectures are pairwise common knowledge between Bob and Claire, between Claire and Donald, as well as between Donald and Alice. However, conjectures are not commonly known. In fact, they are not even mutually known, as Claire does not know Alice's conjecture at $\omega_{1}$ : given her information set $P_{\text {Claire }}\left(\omega_{1}\right)=\left\{\omega_{1}, \omega_{2}\right\}$, she attaches probability of only $\frac{1}{2}$ to Alice being certain that each of her opponents chooses $\ell$.

Furthermore, note that rationality is $G$-pairwise mutual knowledge at $\omega_{1}$. However, it is not mutually known at $\omega_{1}$ that everyone is rational. Indeed, Claire does not know that Alice is rational at $\omega_{1}$, since $\ell$ is not a best response for Alice at world $\omega_{2}$.

Besides, observe that for every $i \in I$, the remaining players share the same marginal conjecture about $i$ 's choice at $\omega_{1}$, i.e. $\operatorname{marg}_{S_{i}} \hat{\mu}_{j}\left(\omega_{1}\right)=\sigma_{i}$ for all $j \in I \backslash\{i\}$, where the probability measure $\sigma_{i}$ assigns probability 1 to $i$ playing $\ell$. Also, $(\ell, \ell, \ell, \ell)$ constitutes a Nash equilibrium of $\Gamma$.

In the preceding example neither the conjectures nor rationality are mutually known. Hence, the two central elements of Aumann and Brandenburger's conditions for Nash equilibrium are violated, yet both of their conclusions do hold. Indeed, players entertain the same marginal conjectures about their opponents' strategies, and also these marginal conjectures form a Nash equilibrium. On the basis of Example 1 the natural question then arises, whether there exists a general relation between $G$ mutual knowledge of rationality and $G$-common knowledge of conjectures on the one hand, and Nash equilibrium on the other hand.

\section{PAIRWISE INTERACTIVE KNOWLEDGE AND NASH EQUILIBRIUM}

We now weaken Aumann and Brandenburger's conditions for Nash equilibrium by means of pairwise interactive knowledge. Indeed, the following result shows that $G$-pairwise mutual knowledge of rationality and $G$-pairwise common knowledge of conjectures already suffice for Nash equilbrium, if $G$ is Hamiltonian.

THEOREM 2 Let $\Gamma$ be a game in normal form, $\mathcal{A}^{\Gamma}$ be an epistemic model of it, and $G$ be a Hamiltonian undirected graph. Suppose that at some world $\omega \in \Omega$ rationality is G-pairwise mutual knowledge and 
there exists a tuple $\left(\mu_{1}, \ldots, \mu_{N}\right)$ of conjectures which is G-pairwise common knowledge. Then, there exists a mixed strategy profile $\left(\sigma_{1}, \ldots, \sigma_{N}\right)$ such that

(i) $\operatorname{marg}_{S_{j}} \mu_{i}=\sigma_{j}$ for all $i \in I \backslash\{j\}$, and

(ii) $\left(\sigma_{1}, \ldots, \sigma_{N}\right)$ is a Nash equilibrium of $\Gamma$.

ProOF: Suppose for sake of simplicity and without loss of generality that the Hamiltonian path in $G$ is $\{1, \ldots, N\}$, i.e. $i_{k}=k$ for all $k=1, \ldots, N$.

Proof of (i). This result is a direct consequence of repeatedly applying Aumann's agreement theorem (Aumann, 1976). First observe that

$$
\begin{aligned}
{\left[\mu_{i}\right] } & =\left\{\omega \in \Omega: \hat{\mu}_{i}(\omega)=\mu_{i}\right\} \\
& \subseteq\left\{\omega \in \Omega: \operatorname{marg}_{S_{j}} \hat{\mu}_{i}(\omega)=\operatorname{marg}_{S_{j}} \mu_{i}\right\} \\
& =:\left[\operatorname{marg}_{S_{j}} \mu_{i}\right],
\end{aligned}
$$

implying that for all $i, k \in I \backslash\{j\}$,

$$
C K_{i, k}\left(\left[\mu_{i}\right] \cap\left[\mu_{k}\right]\right) \subseteq C K_{i, k}\left(\left[\operatorname{marg}_{S_{j}} \mu_{i}\right] \cap\left[\operatorname{marg}_{S_{j}} \mu_{k}\right]\right)
$$

Since, $\operatorname{marg}_{S_{j}} \mu_{i}$ and $\operatorname{marg}_{S_{j}} \mu_{k}$ are posterior probability distributions, in the sense that for each $s_{j} \in S_{j}$,

$$
\operatorname{marg}_{S_{j}} \mu_{i}\left(s_{j}\right)=\pi\left(\left[s_{j}\right] \mid P_{i}(\omega)\right) \text { and } \operatorname{marg}_{S_{j}} \mu_{k}\left(s_{j}\right)=\pi\left(\left[s_{j}\right] \mid P_{k}(\omega)\right),
$$

it follows from Aumann's agreement theorem that $\omega \in C K_{i, k}\left(\left[\operatorname{marg}_{S_{j}} \mu_{i}\right] \cap\left[\operatorname{marg}_{S_{j}} \mu_{k}\right]\right)$ implies

$$
\operatorname{marg}_{S_{j}} \mu_{i}=\operatorname{marg}_{S_{j}} \mu_{k}
$$

Fix some $j \in I$. Then, it follows from the structure of $G$ and repeatedly applying Equation (1) that

$$
\begin{aligned}
\operatorname{marg}_{S_{j}} \mu_{j+1} & =\operatorname{marg}_{S_{j}} \mu_{j+2} \\
& =\cdots \\
& =\operatorname{marg}_{S_{j}} \mu_{N} \\
& =\operatorname{marg}_{S_{j}} \mu_{1} \\
& =\cdots \\
& =\operatorname{marg}_{S_{j}} \mu_{j-1} .
\end{aligned}
$$

Henceforth, for all $i, j \in I$, let $\sigma_{j}:=\operatorname{marg}_{S_{j}} \mu_{i}$.

Proof of (ii). First, we show that for all $E \subseteq \Omega$,

$$
\pi\left(E \cap\left[s_{i}\right] \mid P_{i}(\omega)\right)=\pi\left(E \mid P_{i}(\omega)\right) \cdot \pi\left(\left[s_{i}\right] \mid P_{i}(\omega)\right) .
$$


This follows directly from the fact that $\left[s_{i}\right]$ is $\mathcal{P}_{i}$-measurable: More specifically, if $\hat{s}_{i}(\omega)=s_{i}$, then $P_{i}(\omega) \subseteq\left[s_{i}\right]$, in which case $\pi\left(E \cap\left[s_{i}\right] \mid P_{i}(\omega)\right)=\pi\left(E \mid P_{i}(\omega)\right)=\pi\left(E \mid P_{i}(\omega)\right) \cdot \pi\left(\left[s_{i}\right] \mid P_{i}(\omega)\right)$. Alternatively, if $\hat{s}_{i}(\omega) \neq s_{i}$, then $P_{i}(\omega) \cap\left[s_{i}\right]=\emptyset$. Then, $\pi\left(E \cap\left[s_{i}\right] \mid P_{i}(\omega)\right) \leq \pi\left(\left[s_{i}\right] \mid P_{i}(\omega)\right)=0$.

Now, we show that for all $i \in I$

$$
\mu_{i}(\omega)=\operatorname{marg}_{S_{1}} \hat{\mu}_{i}(\omega) \times \cdots \times \operatorname{marg}_{S_{i-1}} \hat{\mu}_{i}(\omega) \times \operatorname{marg}_{S_{i+1}} \hat{\mu}_{i}(\omega) \times \cdots \times \operatorname{marg}_{S_{n}} \hat{\mu}_{i}(\omega)
$$

Without loss of generality, we show it for player 1 . Observe that for an arbitrary $\left(s_{2}, \ldots, s_{N}\right) \in S_{-1}$,

$$
\begin{aligned}
\hat{\mu}_{1}(\omega)\left(s_{2}, \ldots, s_{N}\right) & =\pi\left(\left[s_{2}\right] \cap \cdots \cap\left[s_{N}\right] \mid P_{1}(\omega)\right) \\
& =\pi\left(\left[s_{2}\right] \cap \cdots \cap\left[s_{N}\right] \mid M_{1,2}(\omega)\right) \\
& =\sum_{P_{2} \subseteq M_{1,2}(\omega)} \pi\left(\left[s_{2}\right] \cap \cdots \cap\left[s_{N}\right] \mid P_{2}\right) \cdot \pi\left(P_{2} \mid M_{1,2}(\omega)\right) .
\end{aligned}
$$

Then, it follows from Equation (2) that

$$
\begin{aligned}
\hat{\mu}_{1}(\omega)\left(s_{2}, \ldots, s_{N}\right) & =\sum_{P_{2} \subseteq M_{1,2}(\omega)} \pi\left(\left[s_{3}\right] \cap \cdots \cap\left[s_{N}\right] \mid P_{2}\right) \cdot \pi\left(\left[s_{2}\right] \mid P_{2}\right) \cdot \pi\left(P_{2} \mid M_{1,2}(\omega)\right) \\
& =\sum_{P_{2} \subseteq M_{1,2}(\omega)} \pi\left(\left[s_{3}\right] \cap \cdots \cap\left[s_{N}\right] \mid P_{2}(\omega)\right) \cdot \pi\left(\left[s_{2}\right] \mid P_{2}\right) \cdot \pi\left(P_{2} \mid M_{1,2}(\omega)\right) \\
& =\pi\left(\left[s_{3}\right] \cap \cdots \cap\left[s_{N}\right] \mid P_{2}(\omega)\right) \sum_{P_{2} \subseteq M_{1,2}(\omega)} \pi\left(\left[s_{2}\right] \mid P_{2}\right) \cdot \pi\left(P_{2} \mid M_{1,2}(\omega)\right) \\
& =\pi\left(\left[s_{3}\right] \cap \cdots \cap\left[s_{N}\right] \mid P_{2}(\omega)\right) \cdot \pi\left(\left[s_{2}\right] \mid M_{1,2}(\omega)\right) \\
& =\pi\left(\left[s_{3}\right] \cap \cdots \cap\left[s_{N}\right] \mid P_{2}(\omega)\right) \cdot \pi\left(\left[s_{2}\right] \mid P_{1}(\omega)\right) \\
& =\hat{\mu}_{2}(\omega)\left(s_{3}, \ldots, s_{N}\right) \cdot \hat{\mu}_{1}(\omega)\left(s_{2}\right) .
\end{aligned}
$$

Repeat the previous step inductively to obtain

$$
\hat{\mu}_{1}(\omega)\left(s_{2}, \ldots, s_{N}\right)=\hat{\mu}_{1}(\omega)\left(s_{2}\right) \cdots \hat{\mu}_{N-1}(\omega)\left(s_{N}\right)
$$

and recall from $(i)$ that all players agree on the marginal conjectures, implying that

$$
\hat{\mu}_{1}(\omega)\left(s_{2}, \ldots, s_{N}\right)=\hat{\mu}_{1}(\omega)\left(s_{2}\right) \cdots \hat{\mu}_{1}(\omega)\left(s_{N}\right)
$$

which proves Equation (3).

Since every player knows their own conjecture, it follows that $\left(\hat{\mu}_{1}(\omega), \ldots, \hat{\mu}_{N}(\omega)\right)=\left(\mu_{1}, \ldots, \mu_{N}\right)$. Recall from (i) that

$$
\left(\sigma_{1}, \ldots, \sigma_{N}\right):=\left(\operatorname{marg}_{S_{1}} \hat{\mu}_{2}(\omega), \ldots, \operatorname{marg}_{S_{N}} \hat{\mu}_{1}(\omega)\right)
$$


For an arbitrary $i \in I$, we are going to show that $s_{i} \in B R_{i}(\sigma)$ for every $s_{i} \in \operatorname{supp}\left(\sigma_{i}\right)$. For every $s_{i} \in \operatorname{supp}\left(\sigma_{i}\right)$ observe that there exists some $\omega^{\prime} \in P_{i+1}(\omega)$ such that $\hat{s}_{i}\left(\omega^{\prime}\right)=s_{i}$. Since $\omega \in K_{i+1}\left(R_{i}\right)$ it follows that $P_{i+1}(\omega) \subseteq R_{i}$, and therefore $\hat{s}_{i}\left(\omega^{\prime}\right) \in B R_{i}\left(\hat{\mu}_{i}\left(\omega^{\prime}\right)\right)$. Finally, it follows from $\omega \in C K_{i, i+1}\left(\left[\mu_{i}\right]\right)$ that $\hat{\mu}_{i}\left(\omega^{\prime}\right)=\hat{\mu}_{i}(\omega)$, and therefore

$$
\begin{aligned}
s_{i} & \in B R_{i}\left(\hat{\mu}_{i}\left(\omega^{\prime}\right)\right) \\
& =B R_{i}\left(\hat{\mu}_{i}(\omega)\right) \\
& =B R_{i}\left(\operatorname{marg}_{S_{1}} \hat{\mu}_{i}(\omega) \times \cdots \times \operatorname{marg}_{S_{i-1}} \hat{\mu}_{i}(\omega) \times \operatorname{marg}_{S_{i+1}} \hat{\mu}_{i}(\omega) \times \cdots \times \operatorname{marg}_{S_{N}} \hat{\mu}_{i}(\omega)\right) \\
& =B R_{i}\left(\sigma_{1}, \ldots, \sigma_{N}\right),
\end{aligned}
$$

which concludes the proof.

Q.E.D.

The contribution of the previous result to the epistemic foundations of Nash equilibrium is twofold.

Firstly, we significantly relax the standard epistemic conditions of Aumann and Brandenburger (1995), by no longer requiring neither common knowledge of conjectures nor mutual knowledge of rationality.

Secondly, Theorem 2 offers further insight on the relation between Nash equilibrium and common knowledge of rationality. In fact, for many years the predominant view suggested that common knowledge of rationality was an essential element of Nash equilibrium. This view was then challenged by Aumann and Brandenburger (1995) who required only mutual knowledge of rationality in their foundation for Nash equilibrium. However, Polak (1999) observed more recently that Aumann and Brandenburger's conditions actually do imply common knowledge of rationality. In a sense, his result thus restored some of the initial belief in the importance of common knowledge of rationality in the context of Nash equilibrium. Our theorem not only confirms Aumann and Brandenburger's initial intuition about the non-necessity of common knowledge of rationality for Nash equilibrium, but also provides sufficient conditions for Nash equilibrium that do not even imply mutual knowledge of rationality. To see this, consider Example 1, and observe that at $\omega_{1}$, which satisfies all the conditions of our theorem, Claire does not know that Alice is rational, implying that $\omega_{1} \notin K_{\text {Claire }}\left(R_{\text {Alice }}\right)$.

\section{DISCUSSION}

Tightness. The assumption of the graph being Hamiltonian is crucial for Theorem 2. Indeed, it is now shown by means of an example that the graph simply being connected does not suffice for the conclusions of Theorem 2 to obtain, even if rationality is common knowledge. In that sense our epistemic foundation is tight.

ExAmple 2 Consider the anti-coordination game $\Gamma=\left(I,\left(S_{i}\right)_{i \in I},\left(U_{i}\right)_{i \in I}\right)$, where $I=\{$ Alice, Bob, 
Claire $\}, S_{i}=\{h, \ell\}$ for all $i \in I$, and

$$
U_{i}\left(s_{\text {Alice }}, s_{\text {Bob }}, s_{\text {Claire }}\right)= \begin{cases}0 & \text { if } s_{\text {Alice }}=s_{\text {Bob }}=s_{\text {Claire }} \\ 1 & \text { otherwise }\end{cases}
$$

Now, suppose that an epistemic model $\mathcal{A}^{\Gamma}$ of $\Gamma$ is given by

$$
\begin{aligned}
\Omega & =\left\{\omega_{1}, \omega_{2}\right\} \text { endowed with a uniform common prior } \pi \\
\mathcal{P}_{\text {Alice }} & =\left\{\left\{\omega_{1}\right\}_{h} ;\left\{\omega_{2}\right\}_{h}\right\} \\
\mathcal{P}_{\text {Bob }} & =\left\{\left\{\omega_{1}\right\}_{h} ;\left\{\omega_{2}\right\}_{\ell}\right\} \\
\mathcal{P}_{\text {Claire }} & =\left\{\left\{\omega_{1}, \omega_{2}\right\}_{\ell}\right\}
\end{aligned}
$$

with the information sets' indices denoting the respective player's strategy given the choice function. Let $G=(I, \mathcal{E})$ be a connected graph such that

$$
\begin{aligned}
I & =\{\text { Alice }, \text { Bob, Claire }\} \\
\mathcal{E} & =\{(\text { Alice }, \text { Bob }),(\text { Bob }, \text { Claire })\}
\end{aligned}
$$

Note that at every $\omega \in \Omega$, rationality is common knowledge, and conjectures are $G$-pairwise common knowledge. Moreover, at $\omega_{1}$, Alice is certain that Bob chooses $h$ and Claire chooses $\ell$, whereas Claire's conjecture attaches probability $\frac{1}{2}$ to both of her opponents playing $h$, and $\frac{1}{2}$ to Alice playing $h$ and Bob playing $\ell$. Therefore, Alice and Claire disagree on their marginal conjecture about Bob's choice, implying that the conclusion of Theorem 2 does not hold. In fact, all conditions of Theorem 2 are satisfied apart from $G$ being Hamiltonian. Hence, $G$ simply being connected instead of Hamiltonian does not suffice for Nash equilibrium.

Knowledge of an opponent's conjecture. Already Aumann and Brandenburger (1995) recognize the conceptual difficulty in assuming knowledge of an opponent's conjecture. We do not intend to provide any remedy to this problematic assumption whatsoever. Indeed, the conceptual issue imposed by assuming knowledge of opponents' conjectures persists. However, we show that less knowledge about the opponents' conjectures is actually needed for Nash equilibrium to obtain.

Related Literature. The sufficient conditions by Aumann and Brandenburger (1995), which we present in Section 2.3 above, have become the standard epistemic foundation for Nash equilibrium. Subsequently, Polak (1999) showed that for complete information games, under Aumann and Brandenburger's assumption of commonly known conjectures, mutual knowledge of rationality does in fact imply common knowledge of rationality. More recently, Perea (2007) derived Nash equilibrium in a one-person perspective epistemic model, and Barelli (2009) proposed a foundation for Nash equilibrium without common 
priors.

Department of Quantitative Economics, Maastricht University

c. bach@maastrichtuniversity.nl

and

Department of Economics, Maastricht University

e.tsakas@maastrichtuniversity.nl

\section{REFERENCES}

Aumann, R.J. (1976). Agreeing to disagree. Annals of Statistics 4, 1236-1239.

Aumann, R.J. \& Brandenburger, A. (1995). Epistemic conditions for Nash equilibrium. Econometrica 63, 1161-1180.

Barelli, P. (2009). Consistency of beliefs and epistemic conditions for Nash and correlated equilibria. Games and Economic Behavior 67, 363-375.

NASH, J. (1951). Non-cooperative games. Annals of Mathematics 54, 286-295.

Perea, A. (2007). A one-person doxastic characterization of Nash strategies. Synthese 158, 251-271.

PolAK, B. (1999). Epistemic conditions for Nash equilibrium, and common knowledge of rationality. Econometrica 67, 673-676. 